\title{
Timpanoplastía en niños: Experiencia de 10 años en el Hospital Clínico de la Universidad de Chile ${ }^{1}$
}

\author{
Tympanoplasty in children: 10 years' experience \\ at the Clinical Hospital of Universidad de Chile
}

Patricia Esquivel $\mathrm{C}^{2}$, Alfredo Naser $\mathrm{G}^{2}$, Carolina Bustamante $\mathrm{Y}^{2}$.

\begin{abstract}
RESUMEN
日 objetivo de este trabajo fue conocer el resultado anatómico y audiológico de las timpanoplastías, evaluar factores pronósticos asociados a la intervención y comparar los resultados con la literatura publicada. Para ello se revisaron las fichas clínicas de los pacientes menores de 14 años intervenidos en el Hospital Cínico de la Universidad de Chile entre 1994 y 2003 en quienes se realizó timpanoplastía, sin mastoidectomía Se incluyeron sólo aquellos pacientes que tuvieron control audiométrico pre $y$ postoperatorio y un seguimiento clínico mínimo de 6 meses postoperatorio.

Se trabajó con un total de 41 oídos operados, consignando edad, sexo, tipo de perforación, tipo de cirugía (vía de abordaje, tipo de injerto y colocación del injerto), resultado anatómico, resultado auditivo y complicaciones postoperatorias.

$\theta$ promedio de edad fue de 8,3 años (rango entre 4 y 14 años). No hubo diferencias según género. $\theta$ éxito anatómico alcanzó el $90 \%$ y la mejoría auditiva fue significativa en $78 \%$ de los casos. No se encontró relación entre la vía de abordaje, tipo de injerto y su técnica de colocación con el éxito postoperatorio. Se observó un mayor porcentaje de éxito anatómico y auditivo en niños menores de 8 años $(p<0,05)$.

Los resultados del estudio son concordantes con la literatura, siendo la edad el único factor que influye en el éxito postquirúrgico. Los mejores resultados se obtuvieron en niños menores de 8 años, siendo este resultado anatómico incluso mejor que los observados en la población general.
\end{abstract}

Palabras claves: Timpanoplastía, miringoplastía, otitis media crónica

\section{SUMMARY}

The objective of this paper was to know the anatomic and audiological results of tympanoplasties, evaluate prognostic factors associated to the surgery and compare the results with published literature. For this purpose the clinical file cards of patients under 14

\footnotetext{
1 Trabajo presentado en la Reunión de la Sociedad Chilena de Otorrinolaringología Medicina y Grugía de Cabeza y Ouello, junio 2004.

${ }^{2}$ Médicos del Servicio de Otorrinolaringología, Hospital Cínico de la Universidad de Chile.
} 
years of age operated at the Cinical Hospital of Universidad de Chile between 1994 and 2003 were reviewed, in whom tympanoplasty without mastoidectomy was practiced. Only those patients who were subject to pre and postoperatory audiometric control were included, and with a minimum clinical follow-up six months postoperatory. Work was conducted with 41 ears operated, registering age, sex, type of perforation, type of surgery (approach passage, type of graft and placement of graft), anatomical result, auditive result and postoperatory complications. The average age was 8,3 years (range between 4 and 14 years). There were no differences by gender. The anatomic result amounted to $90 \%$ and the auditive improvement was significant in $78 \%$ of the cases. No relation was found between the approach passage, type of graft and its placement technique and the postoperatory success. A higher percentage of anatomic and auditive success was observed in children under 8 years of age $(p<0,05)$.

The results of the study agree with the bibliography, where age is the sole factor with an influence on the post-surgical success. The best results were obtained in children under 8 years of age, this anatomical result being even better than those observed for the general population.

Key words: Tympanoplasty, miringoplasty, chronical middle ear otitis.

\section{INTRODUCCIÓN}

Desde la descripción de la timpanoplastía realizada por Wullstein en $1952^{1}$ numerosas técnicas y diversos materiales de injerto se han utilizado para cerrar las perforaciones timpánicas. En 1956 se publicó en la Revista Chilena de Otorrinolaringología la primera revisión sobre indicaciones y técnica de timpanoplastía?.

Por su parte, las timpanoplastías en la edad pediátrica comenzaron a realizarse en los $E U U$. desde $1962^{3}$, desde entonces, múltiples estudios acerca de timpanoplastías en niños reportan un éxito quirúrgico entre $56 \%$ y $94 \%{ }^{4}$. Dentro de los trabajos realizados en nuestro país, en $1988 \mathrm{Caro}^{5}$ notifica una supervivencia del injerto en el $85 \%$ de los casos, y un buen resultado auditivo en cerca del $70 \%$. 日 2005 Zelada $^{6}$ reportó un éxito anatómico del $81,5 \%$ en un estudio realizado en el Hospital Barros Luco Trudeau. Lamentablemente, los criterios de éxito no han sido estandarizados, lo que hace difícil la comparación entre los distintos estudios.

Se han discutido extensamente los factores pronósticos que influyen en el éxito anatómico y auditivo de las timpanoplastías. En forma específica se ha intentado establecer el rol de la edad en el éxito del injerto timpánico, estudiando el resultado de la cirugía en niños y adultos. Con respecto a los niños, el tema de las timpanoplastías ha sido extensamente controversial a través de los años, dado que no existe claridad para determinar cuál es la edad en la cual esta cirugía tiene mayores beneficios.

Sabemos que las perforaciones timpánicas en la edad pediátrica constituyen un impedimento importante en la vida de los niños. La miringoplastía es un procedimiento simple y efectivo para cerrar las perforaciones timpánicas en la mayoría de los casos. Sin embargo, no existe consenso entre los otólogos acerca de cuál es la edad óptima para realizar la intervención. Los principales argumentos para intervenir a edades tempranas son: 1 ) Prevenir la posibilidad de una enfermedad crónica del oído y sus complicaciones; 2) Mejorar la audición para optimizar las condiciones del habla y el desarrollo del lenguaje; 3) Facilitar el desarrollo de actividades acuáticas. Por otra parte, la persistencia de disfunción tubaria, las infecciones respiratorias altas recurrentes y la dificultad técnica, son los principales argumentos de quienes defienden la postura de diferir el procedimiento a edades más tardías, preferentemente mayores de 10 años ${ }^{7}$.

En 1999, un metaanálisis concluye que no hay diferencias en cuanto al éxito de la timpanoplastía 
en relación a la edad de intervención ${ }^{4}$. Motivados por esto, quisimos saber cuáles eran los resultados de las timpanoplastías, en niños, realizadas en nuestro hospital, planteándonos los siguientes objetivos:

1. Evaluar el resultado anatómico y auditivo de las timpanoplastías en niños menores de 14 años.

2. Identificar los factores pronósticos de la timpanoplastía en niños.

3. Comparar nuestros resultados con los publicados en la literatura.

\section{PACIENTES Y MÉTODO}

Se realizó un estudio retrospectivo, de las timpanoplastías realizadas entre 1994 y 2003, a niños menores de 14 años, en el Servicio de Otorrinolaringología del Hospital Gínico de la Universidad de Chile.

Se registraron un total de 41 casos que cumplieron con los siguientes criterios de inclusión:

1. Timpanoplastía primaria con o sin reconstrucción de la cadena osicular, sin mastoidectomía.

2. Control audiométrico pre y postoperatorio.

3. Protocolo quirúrgico completo.

4. Seguimiento clínico mínimo de 6 meses postoperatorio.

Se revisó las fichas clínicas de los pacientes, consignando los datos en un protocolo diseñado para este fin. Se consideró el último control médico registrado en la ficha clínica y la última audiometría
Clasificamos los resultados auditivos como buenos (más de $10 \mathrm{~dB}$ de ganancia sobre el promedio tonal puro (PTP) aéreo preoperatorio), regulares (variación entre $+10 \mathrm{~dB}$ y $-10 \mathrm{~dB}$ del umbral preoperatorio) y malos (más de $10 \mathrm{~dB}$ de pérdida en relación al preoperatorio).

Los datos obtenidos se analizaron estadísticamente con el programa computacional $\boxminus$ IIInfo 6 .

\section{RESULTADOS}

Se incluyeron en el estudio un total de 41 timpanoplastías primarias realizadas a 38 pacientes, de los cuales tres se intervinieron bilateralmente.

En la distribución por edad, el promedio fue de 8,3 años, con un rango entre los 4 y 14 años. En cuanto a la distribución por género no se observan diferencias significativas $52,6 \%$ ( $n=20)$ sexo femenino versus $47,4 \%$ ( $n=18$ ) sexo masculino.

Respecto al número de intervenciones llevadas a cabo por año se pueden observar dos picks, el primero el año 1997 con 6 intervenciones y posteriormente en el año 2002 con 7 cirugías (Fgura 1), en general se puede observar que la indicación de cirugía ha ido incrementándose con el paso de los años, sin observarse diferencias significativas en los últimos 2 años.

En relación con el diagnóstico preoperatorio un $44 \%$ se trataba de Oitis Media Orónica (OMC) bilateral, un 51\% OMC unilateral y dos casos (5\%) correspondieron a perforaciones timpánicas de

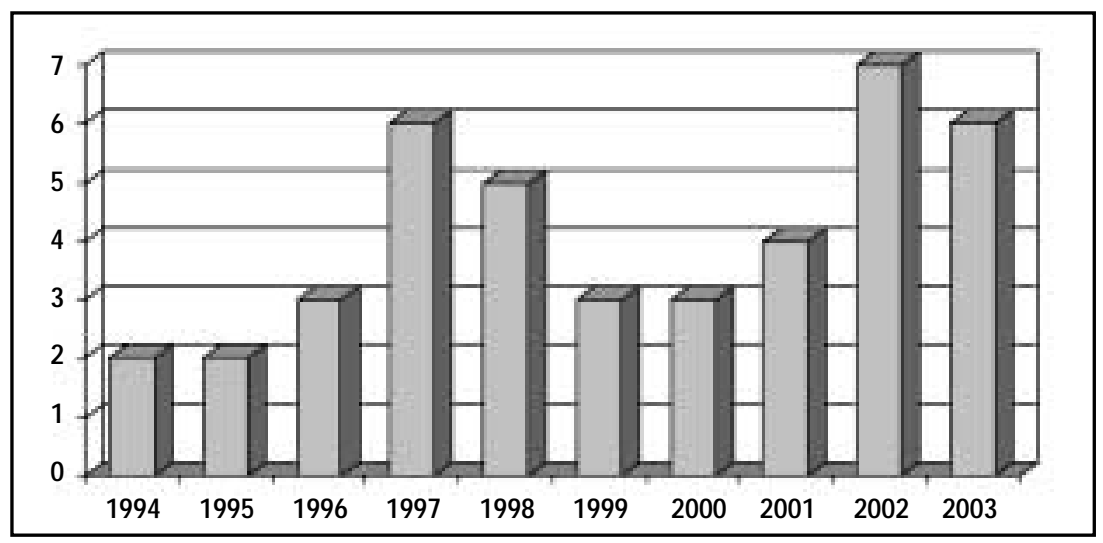

Figura 1. Número de timpanoplastías realizadas por año en el Hospital Cínico de la Universidad de Chile. 
origen traumático, con una evolución mayor a 10 meses sin resolución espontánea, una de ellas secundaria a la colocación de tubo de ventilación. Cabe destacar que en $100 \%$ de los casos las OMC tenían un periodo mayor o igual a seis meses de oído seco previo a la intervención.

Respecto a la técnica quirúrgica, se utilizó la vía de abordaje endoaural en un $68 \%$ de los casos y la retroauricular en el 32\%. 日 tipo de injerto utilizado fue principalmente de fascia en un $42 \%$, siguiéndolo en frecuencia el injerto de tipo compuesto (cartílago pericondrio) con un $24 \%$, solo pericondrio en un $22 \%$ y grasa en un $12 \%$ (Tabla 1). Acerca de la colocación del injerto se prefirió la posición lateral en un $76 \%$ de los casos, significativamente mayor respecto al resto de ubicaciones (Tabla 2).

Tabla 1. Tipo de injerto utilizado

\begin{tabular}{|lr|}
\hline Tipo de injerto & $\%$ \\
\hline Fascia & 42 \\
Cartílago-peric & 24 \\
Pericondrio & 22 \\
Grasa & 12 \\
Total & 100 \\
\hline
\end{tabular}

Tabla 2. Colocación del injerto

\begin{tabular}{|lr|}
\hline Colocación del injerto & $\%$ \\
\hline Lateral & 76 \\
Transtimpánica & 12 \\
Medial & 10 \\
Empalizada & 2 \\
Total & 100 \\
\hline
\end{tabular}

Tabla 3. Resultados anatómicos de timpanoplastías en niños entre 4 y 14 años

\begin{tabular}{|lrr|}
\hline Resultado anatómico & $\mathrm{N}$ & $\%$ \\
\hline Tímpano indemne & 37 & 90 \\
Alteración del injerto & 1 & 2,4 \\
Retracción & 0 & 0 \\
Blunting & 0 & 0 \\
Lateralización & 1 & 2,4 \\
Reperforación & 3 & 7,3 \\
\hline
\end{tabular}

No se encontró relación estadísticamente significativa entre la vía de abordaje, el tipo de injerto y la colocación de éste, con respecto al resultado anatómico final.

En el 95\% se realizó unatimpanoplastíatipo I. Sólo en 2 casos se realizó una timpanoplastía tipo III (4,87\%), ambos casos portadores de una fijación de cadena Cabe destacar que los dos casos de timpanoplastíatipo III presentaron alteración del injerto en el postoperatorio, uno con lateralización del injerto y el otro con una reperforación. La reconstrucción de la cadena osicular fue necesaria solo en un caso (2,4\%).

Los resultados anatómicos fueron muy satisfactorios, encontrándose un neotímpano indemne en el $90 \%$ de los casos. Sólo 3 tímpanos $(7,3 \%)$ presentaron reperforación y en un caso $(2,4 \%)$ se evidenció la lateralización del injerto (Tabla 3).

Para estudiar los resultados según la edad de los pacientes, dividimos la muestra en dos grupos: 日 primer grupo conformado por 20 niños entre 4 y 8 años de edad y el segundo grupo conformado por 21 niños entre 9 y 14 años de edad.

Destaca que al estudiar los resultados anatómicos por edad, se observó un $100 \%$ de éxito cuando la cirugía se realizaba en niños menores de 8 años. A diferencia de lo que ocurría en niños de 9 años o más, en que el éxito anatómico postquirúrgico tan sólo alcanzó un $82 \%$, diferencia que es estadísticamente significativa $(p<0,05)$ (Figura 2$)$.

Por otra parte, los resultados auditivos fueron muy buenos. Se analizaron las diferencias entre el PIP aéreo preoperatorio y PIP aéreo postoperatorio,

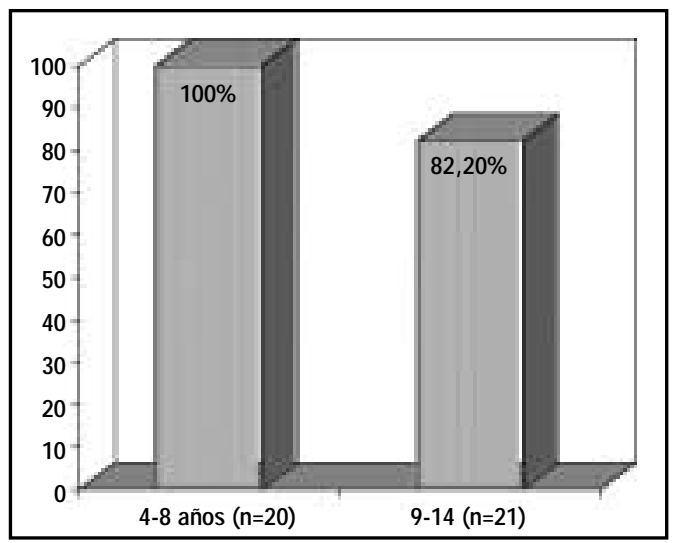

Figura 2. Éxito anatómico según edad $(p<0,05)$. 


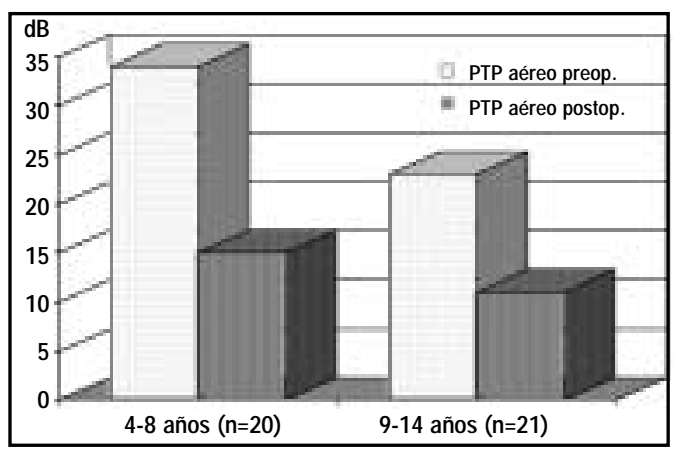

Figura 3. Comparación de PTP aéreo pre y postquirúrgico según edad $(p<0,05)$.

en ambos grupos se pudo observar una mejoría del PIP, observándose una diferencia significativamente mayor en el grupo de 4 a 8 años de edad en que la ganancia entre el PTP pre y postoperatorio fue de 19 $\mathrm{dB}$, mientras que en el grupo de 9 a 14 años la ganancia fue de sólo $12 \mathrm{~dB}$ (Fgura 3).

Del grupo de estudio en su totalidad, clasificamos los resultados auditivos como buenos (más de $10 \mathrm{~dB}$ de ganancia sobre el PTP aéreo preoperatorio) en el $78 \%$ de los casos $(n=32)$, mientras que un $22 \%(n=9)$ presentó entre 0 y 10 $\mathrm{dB}$ de ganancia respecto al PTP preoperatorio (Tabla 4). Destaca que en ningún caso la audición disminuyó después del procedimiento quirúrgico, y que las menores mejorías auditivas se registraron en los 2 casos de timpanoplastía tipo III, ambos sólo con $5 \mathrm{~dB}$ de ganancia en el PTP postoperatorio, y ambos pacientes pertenecientes al grupo de entre 9 y 14 años de edad.

\section{DISCUSIÓN}

Tal como se mencionó anteriormente, aún no existe consenso acerca de cuál sería la edad ideal para realizar la timpanoplastía en niños portadores de una perforación timpánica, pues aún no se tienen claros cuáles serían los factores que tendrían un mayor valor pronóstico en el resultado postoperatorio. Sin embargo, varios estudios coinciden en que factores como una mala función tubaria, junto con la gran cantidad de infecciones respiratorias agudas que se presentan con mayor frecuencia en la edad pediátrica, predisponen a una mayor cantidad de fracasos en el
Tabla 4. Resultados auditivos de timpanoplastías en niños entre 4 y 14 años

\begin{tabular}{|lrr|}
\hline Cierre de Gap & $\mathrm{N}$ & $\%$ \\
\hline$>10 \mathrm{~dB}$ & 32 & 78 \\
$0-10 \mathrm{~dB}$ & 9 & 22 \\
$<0 \mathrm{~dB}$ & 0 & 0 \\
\hline
\end{tabular}

postoperatorio 8,9 . Ya desde 1976, Gasscock menciona que la edad pediátrica sería una "contraindicación relativa" para este procedimiento dada la alta tasa de infecciones respiratorias altas y otitis media aguda ${ }^{10}$. En 1990 Koch et al ${ }^{9}$ reportaron un rango de éxito del $81 \%$ para timpanoplastías en niños mayores de 8 años, sin embargo, esta cifra caía notoriamente en niños menores de 8 años, siendo cercana al 30\%. Son estos autores quienes concluyen que la explica ción más probable en estos niños para el fracaso postoperatorio estaría dado por la mayor cantidad de infecciones respiratorias agudas junto con una deficiente función tubaria Lo mismo concluyeron en el año 1992 Smyth et cols ${ }^{11}$, independientemente del tipo de perforación y material de injerto. En el ámbito nacional, en 2005 Zelada y cols ${ }^{6}$ reportan un éxito anatómico postquirúrgico del $83,8 \%$ para niños entre 7 y 14 años, en comparación al 46,6\% de éxito en niños menores de 7 años, independientemente de la técnica quirúrgica

Pese a lo anterior, otros autores como Lau y Toss $^{12}$ en 1986 no encontraron diferencias significativas en los resultados postoperatorios de niños entre los 2 y 7 años de edad versus el grupo entre 8 y 14 años, más aún, ellos sugieren que la cirugía prevendría la reabsorción de la cadena osicular. Ophir et al 4 en el año 1987 reportaron un $79 \%$ de éxito sin diferencias significativas entre los distintos rangos de edad. Kessler et $\mathrm{al}^{7}$, en 1994 revisaron los resultados de209 timpanoplastías tipo I, concluyendo que en los pacientes de 2 años había un éxito del 75 al 94\% y la edad no debiera considerarse como una contraindicación a la cirugía

Los resultados obtenidos por nuestro estudio no difieren a lo encontrado en la literatura9-13. La vía de abordaje, el tipo de injerto y la colocación de éste, parecen no influir en el éxito quirúrgico. Sólo influye ron notoriamente el tipo de cirugía realizada y la edad 
en que se realizó la cirugía $\mathrm{日}$ éxito anatómico postquirúgico fue mayor en pacientes de menor edad (menores de 8 años), alcanzando un 100\% de indemnidad del neotímpano a los 6 meses postoperatorios, mientras que en los niños de 8 años o más, los resultados anatómicos fueron menos exitosos (82\%) y destacando que en este ultimo grupo se encuentran los dos casos de timpanoplastía tipo III, que corresponden a los casos que tuvieron la más baja mejoría auditiva de toda la serie estudiada

Por lo observado en nuestra experiencia, nos parece razonable plantear que la timpanoplastía es un procedimiento simple y eficaz que se puede realizar con muy buenos resultados en la edad pediátrica Estamos concientes que los niños, principalmentelos menores de 8 años, presentan una mayor incidencia de infecciones respiratorias altas (IRA) que pudiesen afectar el resultado postoperatorio, por lo que nos parece prudente realizar un seguimiento estricto de estos pacientes en el preoperatorio, asegurando un tiempo de oído seco previo a la cirugía de a lo menos 6 meses, con lo cual no existiría contraindicación alguna para realizar timpanoplastías en niños menores. No nos parece lógico mantener solo en observación y en espera de probables complicaciones, a niños que pudiesen tener una alternativa curativa a su patología por sobre el $90 \%$ de los casos, obteniendo con una cirugía a edad temprana un buen resultado tanto anatómico como auditivo después de una timpanoplastía

Fnalmente, cabe destacar que nuestro hospital no es pediátrico, sino que, por el contrario, la pobla ción que atiende es principalmente adulta, por esta razón nuestra casuística de timpanoplastías en niños no es muy numerosa a pesar de un seguimiento de 10 años. Lo anterior nos limita a sacar conclusiones extrapolables a lo que ocurriría en centros deatención pediátrica exclusiva, sin embargo, nos parece que nuestros resultados reflejan lo que vemos a diario en nuestra práctica de otorrinolaringología general.

\section{BIBLIOGRAFÍA}

1. WuLsten HL. Funkttionelle Operattionen im Mittelohr mit Hilfe des Freien Spaltlappen-transplantates. Arch Otorhinolaryngolol 1952; 161: 422-35.

2. MASPETIQ $R$. Indicaciones y técnica de la timpa noplastía (RR). Rev otorrinolaringol 1956; $16: 17$.

3. Caylan R, Titiz A, Falaioni M, Donato GD et al. Myringoplasty in children: factors influencing surgical outcome. Atolaryngol Head Neck Surg 1998; 118: 709-13.

4. VRABEC JT, DESKIN RW, GRADY JJ. Meta-analysis of pediatric tympanoplasty. Arch Otolaryngol Surg 1999; 125: 530-4.

5. CaROJ, Matalón C. Timpanoplastía en niños. Rev Aorrinolaringol Or Cabeza Orello 1988; 58: 22-5.

6. ZiAADAU, ARREGUI R, PALAGOSE Timpanoplastías en niños: Experiencia de 10 años. Rev Otorrinola ringol Or Cabeza Ouello 2005; 65: 92-6.

7. KESSLr A A, POTSIC WP, MARSH RR. Tipe I tympanoplasty in children. Arch Oolaringol Head Neck Surg 1994; 120: 487-90.

8. BAJAJ Y, BAIS AS, MURHERJEE B. Tympanoplasty in children: a prospective study. $J$ Laryngol $O$ 1998; 112: 1147-9.

9. KOCH WM, Friedman EM, Mc GlL TJI, HeAly GB. Tympanoplasty in children: The Boston Children's Hospital experience. Arch Otolaryngol Head Neck Surg 1990; 116: 35-40.

10. Glasscock ME 3d. Contraindications to tympanoplasty II: an exercise in clinical judgment. Laryngoscope 1976; 86: 70-6.

11. SMYTH GDI, Toynbøe Memorial Lecture 1992. Factsand fantasies in modern otology: the ear doctor's dilemme. J Laryngol Ool 1992; 106: 951-6.

12. LAU T, TOS M. Tympanoplasty in children: an analysis of late results. Am J Ool 1986; 7: 55-9.

13. N UMAPATHY, RRC; PJ DEKKE, RRCS. Myringoplasty Is it worth performing in Children? Arch Oolaryngol Head Neck Surg 2003; 129: 1053-5.

Dirección: Dra. Patricia Esquivel C.

Santos Dumont 999. Independencia, Santiago de Chile.

Email: pattyesquivel@terra.cl 